\title{
High phenotypic variability among representative strains of common Salmonella enterica serovars with possible implications for food safety
}

Article

Accepted Version

Abdullah, W. Z. W., Mackey, B. M. and Karatzas, K.-A. G. (2018) High phenotypic variability among representative strains of common Salmonella enterica serovars with possible implications for food safety. Journal of Food Protection, 81 (1). pp. 93-104. ISSN 0362-028X doi:

https://doi.org/10.4315/0362-028x.jfp-17-190 Available at https://centaur.reading.ac.uk/72262/

It is advisable to refer to the publisher's version if you intend to cite from the work. See Guidance on citing.

To link to this article DOI: http://dx.doi.org/10.4315/0362-028x.jfp-17-190

Publisher: International Association for Food Protection

All outputs in CentAUR are protected by Intellectual Property Rights law, including copyright law. Copyright and IPR is retained by the creators or other copyright holders. Terms and conditions for use of this material are defined in the End User Agreement. 


\section{www.reading.ac.uk/centaur}

\section{CentAUR}

Central Archive at the University of Reading

Reading's research outputs online 
High phenotypic variability among representative strains of common Salmonella enterica serovars with possible implications for Food Safety

\section{Research Paper}

Wan Zawiah Wan Abdullah ${ }^{1,2}$, Bernard M. Mackey ${ }^{2}$ and Kimon-Andreas G. Karatzas²*

${ }^{1}$ School of Food Science and Technology, Universiti Malaysia Terengganu, 21030, Kuala Terengganu, Terengganu, Malaysia.

${ }^{2}$ School of Chemistry, Food and Pharmacy, Department of Food and Nutritional Sciences, The University of Reading, PO Box 226, Whiteknights,

Reading RG6 6AP, UK.

Keywords: Salmonella, RpoS, strain variability, lysine decarboxylase, biofilms, acid resistance

* Corresponding author

School of Chemistry, Food and Pharmacy

Department of Food \& Nutritional Sciences,

University of Reading,

Reading

RG6 6AD

UK

Room 3-38

Tel. +44 1183786678

Fax. +44 1189310080

Email address: k.karatzas@reading.ac.uk 


\section{ABSTRACT}

Salmonella is an important foodborne pathogen whose ability to resist stress and survive can vary between strains. This variability is normally not taken into account when predictions are made about survival in foods with negative consequences. Therefore, we examined the contribution of variable phenotypic properties to survival under stress in 10 Salmonella serovars. One strain (S. Typhimurium 10) was intentionally an RpoS-negative however, another one ( $S$. Heidelberg) showed an rpoS mutation rendering it inactive. We assessed an array of characteristics (motility, biofilm formation, bile resistance, acid resistance and colony morphology) showing a major variability between strains associated with a 10- to 19-fold difference between the highest and the lowest strain for most characteristics.

The RpoS status of isolates did not affect variability in the characteristics with the exception of resistance to $\mathrm{NaCl}$, acetic acid, lactic acid, and the combination of acetic acid and salt where the variability between the highest and the lowest strain was reduced to 3.1-fold, 1.7-fold, 2-fold and 1.7fold respectively, showing that variability was significant among RpoS-positive strains. Furthermore, we also found a good correlation between acid resistance and lysine decarboxylase activity showing its importance for acid resistance and demonstrated a possible role of RpoS in the lysine decarboxylase activity in Salmonella. 
Non-typhoidal serovars of Salmonella enterica ssp. enterica are common causes of foodborne illness world-wide and represent a major concern to public health as they are responsible for tens of millions of human cases resulting in more than a hundred thousand deaths worldwide annually (33). These organisms must spend a period of time within the animal or avian host where they are exposed to the extreme acid barrier of the stomach, bile salts, high osmolarity, low oxygen concentrations and competition for nutrients and attachment sites. The long-term success of these pathogens depends on their ability to survive in the gastrointestinal system but also on their ability to persist in the external environment during transmission between hosts (27).

Salmonella has several different stress response systems that allow it to withstand adverse conditions including exposure to low $\mathrm{pH}$, oxidative and osmotic stresses, low temperatures, desiccation, bile acids and antimicrobial peptides $(27,30,34)$. Salmonella is also good at forming biofilms which provide protection against low $\mathrm{pH}$, desiccation, host immune responses and antimicrobial agents. Flagella are involved in biofilm formation but also play several other important roles in survival of Salmonella including chemotaxis and competition for nutrients, adherence to and invasion of gut epithelial cells and binding to cholesterol in gallstones within the gall bladder. Given the wide range of distinct cellular properties involved in competition and survival it would be reasonable to expect that exposure to different stresses would select for variants with phenotypes giving particular advantages for specific environments or conditions and there is now increasing evidence for considerable variation between strains in resistance to heat, acid, desiccation and biofilm formation (19). Currently there are more than 2,500 serotypes while less than 100 account for most human infections (3).

Stress-sensitive rpoS mutants are surprisingly common among natural isolates of Salmonella and Escherichia coli. In E. coli the frequency of these mutants can be explained by a growth advantage under conditions of nutrient limitation and are rapidly selected in aged batch cultures or nutrient limited chemostat cultures $(35,22)$. Therefore, in E. coli there is thus a trade-off between survival potential and nutritional competence (SPANC; 22) which is a powerful mechanism giving rise to phenotypic heterogeneity. This remains to be demonstrated in Salmonella as well. 
Control of foodborne illness requires a thorough knowledge of the properties of food-borne pathogenic microorganisms relevant to their ability to survive in food and the environment. As part of their control strategies food producers and regulatory authorities conduct food safety risk assessments based on the likely numbers of particular pathogens in foods, the likely extent of growth or death in the food chain, the infectious dose and amount of food eaten $(19,20,31)$. Relevant data on survival growth and inactivation may be obtained from the literature, from inoculated pack studies and from predictive modelling suites such as ComBase. Such risk assessment studies can identify critical vulnerabilities in the food chain where control measures would be most effectively applied. It is generally recognised that rates of growth and inactivation are most powerfully influenced by external environmental factors particularly temperature, $\mathrm{pH}$, water activity and the presence of preservatives. However as outlined above, survival may also depend on variations in resistance within strains of the same species. This is likely to be especially important under less extreme conditions and where preservation depends on a combination of mild preservative factors. An understanding of strain variability is thus important in assessing the margins of error in model predictions and in choosing appropriate strains for inoculated pack studies.

\section{MATERIALS AND METHODS}

Bacterial strains and growth conditions. Salmonella strains used in this study (Table 1) were maintained at $-70^{\circ} \mathrm{C}$ in cryovial tubes (Greiner Bio-One Ltd., Stroudwater Business Park, GL10 3SX UK). Cells were transferred on Tryptone Soya Agar (TSA; Oxoid, Basingstoke, UK) supplemented with $0.3 \%$ (w/v) yeast extract (Oxoid, Basingstoke, UK) and $0.1 \%$ sodium pyruvate. Modified Brilliant Green agar (MBGA, CM0329), Tryptone Soya Broth (TSB, CM0129), Maximum Recovery Diluent (MRD, CM0733) were all purchased from Oxoid (Basingstoke, UK) prepared according to the manufacturer's instructions. Low Salt Luria Agar contained $10 \mathrm{~g} / \mathrm{L}$ tryptone, $5 \mathrm{~g} / \mathrm{L}$ yeast extract and $12 \mathrm{~g} / \mathrm{L}$ technical agar. Where indicted Congo Red was added to a final concentration of $0.01 \%(\mathrm{w} / \mathrm{v})$. Lysogeny broth (LB) contained $10 \mathrm{~g} / \mathrm{L}$ tryptone, $5 \mathrm{~g} / \mathrm{L}$ yeast extract and $10 \mathrm{~g} / \mathrm{L} \mathrm{NaCl}$. Motility Test Medium (MTM) contained $2.5 \mathrm{~g} / \mathrm{L}$ agar, $10 \mathrm{~g} / \mathrm{L}$ peptone, $5 \mathrm{~g} / \mathrm{L} \mathrm{NaCl}, 3 \mathrm{~g} / \mathrm{L}$ beef extract 
and $0.05 \mathrm{~g} / \mathrm{L}$ 2,3,5 triphenyltetrazolium chloride. Lysine decarboxylase medium (LDM) contained 5 $\mathrm{g} / \mathrm{L}$ peptone, $3 \mathrm{~g} / \mathrm{L}$ yeast extract, $10 \mathrm{~g} / \mathrm{L}$ lysine monohydrochloride and adjusted to $\mathrm{pH} 4.8$ after autoclaving (2).

Single colonies were obtained by streaking a loopful of material from the $-70^{\circ} \mathrm{C}$ stock onto a TSA plate and were used to prepare replicate working stocks $(0.1 \mathrm{~mL})$. These stocks were grown overnight in Tryptone Soya Broth (TSB; Oxoid, Basingstoke, UK) and maintained in $30 \%$ glycerol at $-20^{\circ} \mathrm{C}$. Cells were resuscitated by inoculating $10 \mathrm{~mL}$ TSB with $0.1 \mathrm{~mL}$ frozen stock and incubating statically at $37^{\circ} \mathrm{C}$ or $6 \mathrm{~h}$. Stationary-phase cultures were prepared by inoculating $0.1 \%(\mathrm{v} / \mathrm{v})$ of the $6 \mathrm{~h}$-culture into $20 \mathrm{~mL}$ TSB in a $50 \mathrm{~mL}$ conical flask and incubating with shaking for $18 \mathrm{~h}$ at $37^{\circ} \mathrm{C}$.

Estimation of viable counts. Samples were 10-fold serially diluted in Maximum Recovery Diluent (MRD; Oxoid, Basingstoke, UK) and $0.02 \mathrm{~mL}$ volumes were spread in duplicate on TSA Plates which were incubated at $37^{\circ} \mathrm{C}$ for $24 \mathrm{~h}$. Viability was assessed by counting the number of colonies for each strain and expressing the numbers as $\log (\mathrm{cfu} / \mathrm{mL})$. All experiments were performed at least three times. The loss of viability of each strain was calculated as log reduction.

Survival experiments. Survival was monitored in Nutrient Broth (NB) containing (a) $20 \mathrm{mM}$ sodium acetate adjusted to $\mathrm{pH} 4.0$ with $\mathrm{HCl}$, (b) $20 \mathrm{mM}$ lactic acid pH 3.5 (c) $9.0 \% \mathrm{NaCl}$ and (d) 20 $\mathrm{mM}$ sodium acetate $\mathrm{pH} 4.0$ with $9 \% \mathrm{NaCl}$. Ten $\mathrm{mL}$ of each medium in $25 \mathrm{~mL}$ Sterilin Universal tubes (Thermo Fischer Scientific, Basingstoke, UK) were inoculated with $0.1 \mathrm{~mL}$ of an overnight culture to give an initial cell concentration of $10^{7} \mathrm{CFU} / \mathrm{mL}$. The tubes were stored at $25^{\circ} \mathrm{C}$ and samples were removed at zero time and at appropriate intervals thereafter to assess survival.

Lysine decarboxylase activity. Salmonella cells incubated under acid conditions can raise the $\mathrm{pH}$ of the external medium due to the activity of lysine decarboxylase. Overnight (18 h) cultures grown in LB broth $\left(10^{9} \mathrm{CFU} / \mathrm{mL}\right)$ were centrifuged $(10,000 \times \mathrm{g}$ for $5 \mathrm{~min})$ and resuspended in an equal volume lysine decarboxylase medium (LDM) containing $5 \mathrm{~g} / \mathrm{L}$ peptone, $3 \mathrm{~g} / \mathrm{L}$ yeast extract and $10 \mathrm{~g} / \mathrm{L}$ lysine monohydrochloride which was adjusted to $\mathrm{pH} 4.8$ after autoclaving (2). One and a half 
$\mathrm{mL}$ of this cell suspension containing $\sim 10^{9} \mathrm{CFU}$ was inoculated into $18 \mathrm{~mL}$ LDM in a wide-mouthed Universal bottle. $\mathrm{pH}$ measurements were made at interval up to $5 \mathrm{~h}$. Curves were fitted to plots of $\mathrm{pH}$ versus time using DMFit program available from the ComBase website (http://www.combase.cc) and the time taken for $\mathrm{pH}$ to increase by 0.5 units determined by interpolation.

Testing RpoS phenotype. The bacterial strains were tested for glycogen accumulation and catalase activity. Strains were streaked onto Lysogeny Broth (LB; Oxoid, Basingstoke, UK) agar plates and incubated at $37^{\circ} \mathrm{C}$ for $24 \mathrm{~h}$ then left at $4^{\circ} \mathrm{C}$ for $24 \mathrm{~h}$ before being flooded with iodine solution to detect the level of glycogen (22). Dark brown colonies were presumptive wild-type and white pale brown or white colonies indicated loss of rpoS activity with different levels of glycogen. Catalase activity was examined by transferring a single colony to a glass slide and flooding with $3 \%$ hydrogen peroxide. A catalase-positive strain showed vigorous bubbling.

Colony morphology. Cells from an $18 \mathrm{~h}$ culture in TSB were inoculated into Modified Brilliant Green Agar (MBGA; Oxoid, Basingstoke, UK) by using a sterile needle. Plates were incubated at $20^{\circ} \mathrm{C}$ for $5 \mathrm{~d}$ and their colony morphologies were observed. Low Salt Luria agar plates (tryptone $10 \mathrm{~g} / \mathrm{L}$, yeast extract $5 \mathrm{~g} / \mathrm{L}$, technical agar $12 \mathrm{~g} / \mathrm{L}$ ) containing $0.001 \%$ Congo Red were inoculated with $1 \mu \mathrm{L}$ of $18 \mathrm{~h}$ culture and incubated at $25^{\circ} \mathrm{C}$ or $37^{\circ} \mathrm{C}$ for up to $5 \mathrm{~d}$.

Biofilm Formation. Ten $\mathrm{mL}$ of Low Salt LB in a $25 \mathrm{~mL}$ Universal bottle was inoculated with one loopful of overnight culture and incubated with or without shaking at $20^{\circ} \mathrm{C}$ or $25^{\circ} \mathrm{C}$ for $4 \mathrm{~d}$. Pellicle formation was visually observed before discarding the culture medium. Biofilm adhering to the glass was recorded before and after staining with $0.1 \%(\mathrm{w} / \mathrm{v})$ aqueous crystal violet solution. The following quantitative method was used to test biofilm formation on polystyrene plastic (23): 200 $\mu l$ of overnight culture culture were put into 96 microwell plates (flat bottom) together with a blank and a control (LB broth without salt). The 96 flat bottom microplates were incubated at $25^{\circ} \mathrm{C}$ without shaking. After $4 \mathrm{~d}$ the the liquid cultures were discarded and the plate that has been used for biofilm 
formation was washed twice with distillled water using multi pipette. Plates were inverted on a paper towel to remove excess moisture. Plates were then allowed to air dry in laminar flow hood. Biofilm formation of ten different strains and serovars of Salmonella were stained using $0.1 \%$ aqeous crystal violet stain and were left for about 20 minutes. Stains were discarded and plates were washed again using multipipette with distilled water. Plates were then submerged in bath of distilled water twice and water has been replaced between washes. Plates were tapped on a paper towel to remove excess moisture and were allowed to air dry under the laminar flow hood. The adherent stained biofilms were dissolved with $95 \%$ ethanol and the optical density was measured at $620 \mathrm{~nm}\left(\mathrm{OD}_{620 \mathrm{~nm}}\right)$ at one to two min in a Tecan Sunrise microplate reader (Tecan UK Ltd, Theale, RG7 5AH UK) operated by Magellan software. The higher the reading reflected a stronger biofilm formation.

Motility Test. Cells from an $18 \mathrm{~h}$ culture in LB were inoculated by stabbing with a wire into the centre of the plate containing Motility Test Medium (MTM). In brief, MTM medium contained $2.5 \mathrm{~g} / \mathrm{L}$ agar (Oxoid, Basingstoke, UK), $10 \mathrm{~g} / \mathrm{L}$ peptone, $5 \mathrm{~g} / \mathrm{L} \mathrm{NaCl}$ (Merck, Darmstadt, Germany), 3 $\mathrm{g} / \mathrm{L}$ beef extract (LAB LEMCO) and $0.05 \mathrm{~g} / \mathrm{L} \mathrm{2,3,5}$ triphenyltetrazolium chloride (Sigma-Aldrich, Steinheim, Germany). Plates were incubated at $30^{\circ} \mathrm{C}, 37^{\circ}$ or $15^{\circ} \mathrm{C}$. The distance that the cells had migrated through the semi-solid medium was recorded as the diameter of the area of growth measured in two directions at right angles. The test was repeated on two separate days.

Sensitivity to bile salts. Lysogeny broth was supplemented 0.5\%-5.0\% sodium deoxycholate (Sigma-Aldrich, Gillingham, Dorset,) and adjusted to $\mathrm{pH}$ 7.0. To avoid gel formation by sodium deoxycholate (DOC) this was done in a warm room at $37^{\circ} \mathrm{C}$. The media were then inoculated from an $18 \mathrm{~h}$ culture and $200 \mu \mathrm{l}$ volumes transferred into 96 microwell plates. The plates were then transferred to a Tecan Sunrise Microplate Reader (Tecan UK Ltd., Theale, UK) and optical density was recorded every $20 \mathrm{~min}$ at $620 \mathrm{~nm}$ under static conditions at $37^{\circ} \mathrm{C}$ using the Magellan data analysis software.

Sequencing the $\boldsymbol{r p o S}$ gene. Template DNA was extracted from cultures by using Instagene Matrix (Bio-Rad) according to the manufacturer's instructions. The rpoS gene was amplified using 
primers RPOS-5 (5'-CGG AAT TCT TAT TAT CAT CAA ACA TAA C- 3') and RPOS-3 (3'-CCT TGC CCG GGC TGT GCC GAT GCA C -3'), (13). The primers were synthesized by Sigma-Aldrich and PCR reactions were performed on a Bio- Rad MJ Mini Personal Thermal Cycler (Bio-Rad Laboratories Ltd., Hemel Hempstead, Hertfordshire. PCR products were separated by electrophoresies on a $2 \%(\mathrm{w} / \mathrm{v})$ agarose gel containing ethidium bromide $(5 \mu \mathrm{L}$ per $30 \mathrm{~mL}$ gel). Electrophoresis was performed for 45 minutes at $100 \mathrm{~V}$. Gels were visualized under UV and PCR products were extracted from the gel and cleaned using the MinElute PCR Purification Kit (Qiagen UK, Crawley, UK) according to the manufacturer's instructions. Sequences were generated from the amplified PCR products via Source Bioscience, Department of Biochemistry, University of Oxford.

Data analysis. All experiments described were performed in triplicate. Survival and growth curves were fitted to survival data of log viable count versus time using the DMFit program (freely available at http://www.combase.cc) and times needed to achieve a 1000-fold (3-log) reduction in viable cell concentration were calculated. Optical density growth curves were also analysed using DMFit to obtain lag times and times for an increase of 0.2 OD units. Furthermore, DMFit was also used to analyse curves depicting the $\mathrm{pH}$ increase representing the lysine decarboxylase activity and the biofilm formation. Data from replicate experiments on motility and sensitivity to sodium deoxycholate were analysed with the use of One way ANOVA while Spearman's Rank Correlation Coefficient. was used to rank strains in all survival and motility experiments. The statistical programs used in this study were SPSS version 20 for One way ANOVA and Microsoft Excel for Spearman's Rank Correlation Coefficient. Furthermore, Principal component analysis (PCA) was carried out including all data and data only on stress resistance using the XLSTAT software (https://www.xlstat.com/en/solutions/features/principal-component-analysis-pca).

\section{RESULTS}


Colony morphology. On modified brilliant green agar, all strains showed the typical rdar phenotype exhibited by $S$. Typhimurium 30, with the exception of $S$. Hadar and $S$. Enteritidis 466 which had smooth colonies similar to that of the two RpoS negative strains Typhimurium 10 and Heidelberg (Table 2).

On Congo-red agar, five of the strains namely $S$. Anatum, $S$. Enteritidis 496, $S$. Montevideo, $S$. Typhimurium 30 and $S$. Virchow stained red with a typical rdar phenotype while $S$. Heidelberg and the known RpoS-negative $S$. Typhimurium 10 having smooth, circular pale straw-coloured colonies (Fig. 1). Serovar Hadar colonies were red but much smoother while serovar Newport colonies were smooth and pink after 3 days and developed the complete rdar phenotype after 5 days. Serovar Enteritidis 466 was also smooth and pink after 3 days but developed a rougher 'pimply' appearance after 5 days.

Testing the RpoS phenotype of representative strains from different Salmonella enterica serovars. The RpoS status of the strains was assessed by testing for catalase activity and glycogen accumulation in colonies grown on LB agar. Two strains of known RpoS status were included in the study as references. These were Salmonella serovar Typhimurium strains 30 and 10 which are RpoS positive and negative respectively. The presumptive RpoS status is shown in Table 3. The Salmonella serovar Typhimurium strains of known RpoS status showed the expected phenotype. All other strains were phenotypically RpoS positive except serovar Heidelberg which showed an RpoS negative phenotype.

Sequencing the rpoS genes. Sequencing the $r p o S$ genes of the strains revealed that $S$. Typhimurium strain 10 had $\mathrm{G}$ to $\mathrm{T}$ transversion at nucleotide number 99 changing the GAG triplet codon specifying glutamic acid to a TAG amber nonsense stop codon at position 33 in the amino acid sequence of the protein as described previously (13). Furthermore $S$. Heidelberg had an insertion of a $\mathrm{T}$ at nucleotide number 640 causing a +1 frameshift mutation leaving intact only the first 213 amino acids in the sequence of the protein (Fig. 2). 
Motility. The motility of the test strains was measured as the distance they migrated in sloppy agar in $12 \mathrm{~h}$ at $30^{\circ} \mathrm{C}$ (Fig. 3). Serovar Enteritidis 466 was significantly less motile than Typhimurium 30, Anatum, Hadar and Enteritidis 496 when analysed by ANOVA $(\mathrm{p}<0.05)$. There was some variability between replicate trials but the relative rank order in replicate trials at the same temperature was significantly similar when assessed by Spearman's rank order correlation coefficient $(\mathrm{p}<0.01)$. Experiments at $15^{\circ} \mathrm{C}$ and $37^{\circ} \mathrm{C}$ did not reveal any differences in the rank order of motility as it was similar to that at $30^{\circ} \mathrm{C}$ (Table 4). S. Enteritidis (466) was significantly different at $30^{\circ} \mathrm{C}$ from $S$. Typhimurium (30), $S$. Anatum, $S$. Hadar and $S$. Enteritidis (496) at $\mathrm{p}<0.05$. In addition, the overall rank order shows that serovar Enteritidis (466) migrated at comparatively slower rate followed by $S$. Montevideo and $S$. Heidelberg. S. Montevideo was consistently the second slowest serovar at $30^{\circ} \mathrm{C}$ and $37^{\circ} \mathrm{C}$ and the slowest at $15^{\circ} \mathrm{C}$. Meanwhile, $S$. Enteritidis (466) was the slowest serovar at $30^{\circ} \mathrm{C}$ and S. Heidelberg at $37^{\circ} \mathrm{C}$. Overall there was strain variability with the strain with the highest motility being 2.7-fold more motile than the slowest. This variability was not affected by the RpoS status of the strains.

Biofilm formation. Biofilm formation on glass was measured qualitatively by incubating strains in LB broth without sodium chloride at $20^{\circ} \mathrm{C}$ with shaking. Five of the ten test strains consistently formed good biofilms on the glass surface (Fig 4). Biofilm was absent in the two RpoS negative strains Salmonella Heidelberg and Salmonella Typhimurium strain 10 and was variable in serovars Anatum, Enteritidis 466 and Enteritidis 496. Serovars that showed good biofilm formation also produced obvious pellicles on the liquid surface and showed pronounced sedimentation of cells in the culture. Cultures of RpoS-negative strains had uniform turbidity and showed no sedimentation. Biofilm formation was also assessed after growth in polystyrene microwell plates (see Methods). Table 5 shows the mean optical densities of stained biofilms. The results were broadly similar to those obtained after growth in glass bottles with weak or no biofilm formation in serovars Heidelberg, Typhimurium 10 and Enteritidis 466 . There was significant variability between strains with the strain ranking first producing 15 -fold higher biofilm than the last and this variability was significantly 
affected, although not entirely by the RpoS status, as the RpoS positive $S$. Enteritidis 466 was the weakest biofilm producer.

Sensitivity to sodium deoxycholate. Sensitivity to DOC was assessed by monitoring growth in LB containing concentrations of DOC between $0 \%$ and 5\%. All strains showed growth in both replicates up to $2 \%$ DOC but above this concentration, growth became increasingly variable (Table 6). Salmonella Virchow failed to grow at DOC concentrations above 3\% whereas serovars Anatum and Hadar showed growth in both replicates at all concentrations up to 5\% DOC.

The effect of DOC on the kinetics of growth is illustrated in Fig. 5 with Salmonella Hadar as an example. As the concentration of DOC increased lag times also increased progressively whilst the final biomass reduced. It should be stated that since we measure OD and not CFU, the cells might start growing when their growth is below the detection limit of the spectrophotometer. This could result in recording as a lag phase all the period until the number of cells reach the detection limit and only a part of it might comprise the actual lag phase. However, for the purposes of comparison we will call this period as "lag phase" although this might not be entirely accurate.

Mean lag times increased 10-fold from about 100 min to just below 1000 min as DOC increased from $0 \%$ to $5.0 \%$ but the increase was gradual rather than biphasic (data not shown). To allow comparisons between strains over a range of concentrations, individual lag times were normalised by dividing individual lags by the mean lag. The resultant ratios were compared at DOC concentrations between 0.5 and $2.0 \%$ - this being the maximum concentration where growth was recorded in all strains in both replicates (Table 7). Analysis of lag ratios by ANOVA showed that Salmonella Virchow had significantly longer lags than those of the other strains $(\mathrm{p}<0.05)$. There was significant variability between strains regarding their sensitivity to sodium deoxycholate which was not affected by the inclusion of the RpoS-negative strains with the strain ranking first being 15 -fold more resistant than the last.

Survival studies in Model broth systems. Survival of Salmonella serovars was examined under several conditions. The two RpoS negative strains survived poorly at $\mathrm{pH} 4$ with $20 \mathrm{mM}$ sodium 
acetate (Fig. 6) and in $20 \mathrm{mM}$ lactic acid pH 3.5 (data not shown). There was considerable variability in the acid resistance between strains, as seen after 15 days the difference in the numbers between the most sensitive strain and the most resistant was 3.7 logs ( 5,011-fold) or, 1.7 logs ( 50 -fold $)$ after exclusion of the RpoS positive strains. The kinetics of inactivation were not classical log-linear and as the time of the experiment passed the sensitivity of many strains decreased. Therefore we fitted curves with DMFit using the model that gave the best fit for each curve (see Methods). To compare survival between serovars, curves were fitted with the use of DMFit program and the time required for a $3 \log$ reduction of the viable counts (3 D-value reduction) was calculated (Fig. 7) and the rank order was calculated for each strain (Table 8). A similar analysis was performed in the case of lactic acid treatment (data not shown). The two $S$. Enteritidis strains were ranked equal first with Hadar for resistance to acetic acid and equal second for resistance to acid plus salt. $S$. Hadar was ranked most resistant to salt alone. The two RpoS negative strains were consistently the most sensitive to all three stresses. Spearman's Rank Order Correlation Coefficient was used to determine whether the relative order of resistance was significantly similar in replicate trials of the same stress condition. The results showed that replicate rank orders were significantly similar for acid $(\mathrm{p}<0.002)$, salt $(\mathrm{p}<0.001)$ and acid plus salt $(\mathrm{p}<0.01)$. Taking in account the time for the viable numbers to reduce by $3 \operatorname{logs}$ the variability was significant as the most resistant strain was 10-fold, 4-fold and 19-fold more resistant than the weakest for salt, acetic acid and lactic acid (Fig. 7A) stress respectively. Variability was reduced when we excluded the RpoS-negative strains with the most resistant strain being 3.1-fold, 1.7-fold and 2-fold more resistant than the weakest for salt, acetic acid and lactic acid (Fig. 7A) stress respectively. With the combined stress (acetic acid + salt) the most resistant strain was 10-fold more resistant than the weakest and this difference was reduced to 1.7-fold, when the RpoS-negative strains were excluded.

Whole cell lysine decarboxylase activity. The lysine decarboxylase activity of the serovars was measured as the time taken for the $\mathrm{pH}$ of the $\mathrm{LD}$ medium to increase by 0.5 units due to the lysine carboxylase activity of the cells (from $\mathrm{pH} 4.8$ to $\mathrm{pH} 5.3$ ). While no increase in $\mathrm{pH}$ was seen in the absence of lysine (data not shown), when lysine was present the $\mathrm{pH}$ increased rapidly from $\mathrm{pH} 4.8$ to 
6.3 within 300 min for all RpoS-positive serovars (Fig. 8). However, the two RpoS-negative serovars were significantly slower than the RpoS-positive, raising the $\mathrm{pH}$ about 0.5 units within the first 300 min compared to more than $1.5 \mathrm{pH}$ units for the RpoS-positive serovars. Interestingly, both RpoSnegative serovars were eventually able to neutralise the $\mathrm{pH}$ to 6.5 after 1440 minutes (24 hours). Subsequently we investigated the role of the lysine decarboxylase system in acid resistance of Salmonella by correlating lysine decarboxylase activity expressed as time required for a 0.5 increase of the $\mathrm{pH}$ and acid resistance expressed as the time taken for viable numbers to decrease by $3 \operatorname{logs}$ in the presence of $20 \mathrm{mM}$ lactic acid pH 3.5 (Fig. 9A) or $20 \mathrm{mM}$ acetic acid at pH 4.0 (Fig. 9B). Analysis showed a significant correlation between acid resistance and lysine decarboxylase activity (Fig. 9; $\mathrm{R}^{2}=0.90$ and 0.78 for lactic and acetic acid respectively). Investigation of the RpoS-positive strains only, resulted in a correlation of $\mathrm{R}^{2}=0.40$ and 0.55 for the lactic and acetic acid respectively. This correlation is also reflected in the PCA analysis (Fig. 10) as the lysine decarboxylase activity is exactly antipodal to the acid resistance.

\section{DISCUSSION}

Intra-species variability of microbial growth kinetic behaviour is a feature with vital implications for food safety study (18). The ability of foodborne pathogens to resist and survive stresses can vary between strains of the same species. Although strain variability in terms of response to specific stresses has been well-documented there is much less data on variability in resistance to combined stresses. In this study we investigated the general phenotype of 10 different Salmonella strains, of which 8 were randomly selected, against acid, salt and a combined treatment as well as at the mechanisms that could possibly affect this variability.

First we assessed the colony morphology of the isolates and we observed that all showed the rdar phenotype even in a delayed fashion on both media used, with the exception of $S$. Typhimurium $10, S$. Hadar, $S$. Enteritidis 466 and $S$. Heidelberg which showed smooth colony morphology (Table 2; Fig. 1). The rdar phenotype in Salmonella is known to be affected by LPS structure and curli fimbriae 
production (14). RpoS plays an important role in the development of this phenotype mainly via the control of the expression of agf genes encoding for the thin aggregative fimbriae (32) and loss of RpoS function leads to loss of this phenotype. Therefore, we assessed the phenotypic RpoS status of all strains and found that all were positive except $S$. Typhimurium 10 and $S$. Heidelberg (Table 3). Subsequently, through sequencing we confirmed the previously-described mutation in rpoS of $S$. Typhimurium 10, resulting in an early stop codon (13). In addition, we found that the $S$. Heidelberg strain that was randomly selected, harboured an insertion in its rpoS sequence, resulting in a +1 frameshift mutation and complete loss of sequence after the 214th codon (Fig. 2). However, the reason for the loss of rdar phenotype in $S$. Hadar, $S$. Enteritidis 466 is unknown, but other mutations in genes other than rpoS involved in this phenotype could have such an effect.

Subsequently, we assessed the motility of the different serovars, in different temperatures and all serovars were motile under all conditions while RpoS seemed to play no role in this characteristic (Fig. 3). Overall temperature seemed to affect motility although it did not seem to affect the rank order between strains. Variability was significant with the strain with the highest motility being 2.7fold more motile than the slowest.

Furthermore, we looked at biofilm formation of all strains. Both RpoS-negative were also negative in biofilm formation which is expected as RpoS is known to affect biofilm formation in Salmonella (21, 8) (Fig. 4, Table 5). RpoS, together with other response regulators relays signals from the extracellular environment to the master regulator of biofilm formation, curli subunit gene $\mathrm{D}(\operatorname{csg} D)$ (7). Interestingly, S. Enteritidis (466) despite being an RpoS positive, it was the weakest strain in biofilm formation although it is not known what is the genetic basis of this phenotype in this serotype. We saw great variability in this characteristic which was not affected by the RpoS as the strain with the strongest biofilm production formed 15 -fold more biofilm than the weakest.

Generally, Gram-negative bacteria including Salmonella are resistant to bile. We found that higher concentrations of sodium deoxycholate are inhibitory to the growth of Salmonella (Fig. 5). Interestingly, increasing concentrations of bile salts resulted in a proportional increase of the lag phase and decrease of the final biomass. $S$. Hadar and $S$. Anatum were the most resistant serovars as they grew under all concentrations of bile salts $(0.5-5.0 \%)$. Normally resistance to bile salts is associated 
with long O-Ag polysaccharide chain production such as LPS modifications (16, 25), efflux systems (e.g. multidrug efflux systems; 25) and the SOS response (24). Overall S. Virchow, was the most sensitive to bile salts (Table 5, 6). This might suggest that this serovar is sensitive to bile however, many previous reports have shown its presence in the gall bladder which suggests the opposite (4). RpoS is known to be involved in resistance to bile as it is activated during exposure to deoxycholate, while rpoS mutants are sensitive to bile (10). Furthermore, many RpoS-dependent genes; osm $Y, d p s$, $u s p B$ and $e c n B$ are highly upregulated by deoxycholate in exponential phase (29). However, in our study we did not find major differences between RpoS-positive and RpoS-negative serovars in sensitivity to bile salts. This does not mean that RpoS does not affect bile tolerance in each strain, but that its effect might not be such that could influence the comparison between different strains. This might be due to the great variability between strains as the difference between the most and the least bile resistant strain was 15 -fold and it was not affected by the RpoS status.

From all strains assessed, $S$. Heidelberg and $S$. Typhimurium (10) were consistently the most sensitive to $\mathrm{NaCl}$, acidic conditions and in the combined stress. The similarity between these stresses might have to do with the role of RpoS in both stresses and is demonstrated by the fact that the rank order was similar for acid, salt and acid plus salt and by the PCA analysis (Fig. 10). The two $S$. Enteritidis strains were the most acid resistant, $S$. Hadar was the most salt resistant while S. Virchow was the most resistant in the combined stress (Fig. 6, 7; Table 8). The sensitivity of $S$. Heidelberg and $S$. Typhimurium 10 to both stresses could clearly be attributed to their mutated rpoS genes. RpoS regulates the expression of more than 500 genes of which a significant number is stress genes (9) such as $a d a$ and $y d c l$ that are involved in acid resistance $(11,12)$. The variability was also significant in this case, as the most resistant strain was 10-fold and 4-fold more resistant than the weakest for salt and acid stress respectively, while as expected, it reduced significantly when RpoS-negative strains were excluded. The reduced variability following the exclusion of the RpoS-negative strains was expected due to the major role RpoS plays in salt and acid stress.

Subsequently we investigated the contribution of the lysine decarboxylase system in the variability in acid resistance between Salmonella strains. The lysine decarboxylase is an important factor contributing in the acid resistance of Salmonella $(1,5)$ that can also affect the passage through the 
stomach (17) and its survival in macrophages (28). We found a great variability in the lysine decarboxylase activity between the serovars (Fig. 8) which overall was the result of different lag times in the implementation of the system. However, once the system was active, all strains seemed to possess a similar lysine decarboxylase activity (Fig. 8). Interestingly, the two most acid sensitive and RpoS negative serovars showed the weakest lysine decarboxylase activity (Fig. 8) suggesting that the lysine decarboxylase could be under the control of RpoS. The weaker lysine decarboxylase activity in these two RpoS negative strains was the result of significantly longer lag times resulting in a slow implementation of the system. Similarly to E. coli, most Salmonella enterica strains possess two lysine decarboxylase systems of which, one ( $c a d C A B$ operon) is inducible (26), while the other ( $l d c C$ gene) is regulated by RpoS and it is constitutively expressed in the stationary phase (15). Although there is hardly any published work on the regulation of these systems in Salmonella, if we assume that regulation is similar to $E$. coli, the two RpoS mutants most probably lack $l d c C$ expression and solely rely on the implementation of the inducible system ( $c a d C$, $c a d B, c a d A$ operon) which is slow resulting in extended lag times.

Our analysis showed a good correlation between acid resistance and lysine decarboxylase activity for both for lactic and acetic acid (Fig. 9). This is also reflected in the PCA (Fig. 10) analysis with lysine decarboxylase activity being in the antipodal of acid resistance as longer times recorded at the lysine decarboxylase test correspond to shorter times to $3 \log$ reduction and therefore, weaker strains. This demonstrates the dominant role of the lysine decarboxylase system on the acid resistance in Salmonella. As RpoS affects the expression of various stress genes involved in acid resistance, we performed an analysis including only RpoS-positive strains. This resulted to a reduced but moderate correlation between acid resistance and lysine decarboxylase activity. All these suggest that the lysine decarboxylase system is a major determinant of acid resistance in Salmonella and to a major extend, acid resistance of Salmonella depends on lysine decarboxylase activity.

Our work shows that there is a significant variability between Salmonella serovars in survival towards stresses and other phenotypic characteristics that some are linked to survival and virulence. This high variability is demonstrated by the difference of 15- and 10-fold between the two extreme strains with the exception of acid resistance which was 4-fold. The high variability was not due to the occurrence 
of an RpoS-negative strain (1 out of 8 ) as it affected only the resistance against acid, salt and the combination of the latter two. This is reflected by the PCA analysis where the two RpoS-negative strains, together with $S$. Typhimurium 30 and $S$. Virchow are distinctive but they do not cluster together (Fig. 10A). However, when we performed a PCA analysis including only stress-related data the RpoS-negative strains cluster together far from the main cluster of strains which in this case includes $S$. Typhimurium 30 but not $S$. Virchow which remains distinctively different from all other strains (Fig. 10B).

It should be stated that the $S$. Heidelberg isolate was randomly selected without knowing its RpoS status or its phenotype and its exclusion does not give a good representation of the phenotypic variability in Salmonella. Comparisons excluding this strain are presented here just to understand the contribution of RpoS in phenotypic variability. The inclusion of this RpoS-negative strain is also important because of the common occurrence of such strains reaching significantly high rates in Salmonella and E. coli (13). According to the Self Preservation and Nutritional Competence (SPANC) hypothesis demonstrated in E. coli, RpoS-negative strains have a growth advantage over RpoS positive strains as the lack of expression of a significant number of stress genes allows them to utilise nutrient sources more efficiently towards growth (6). Therefore, the common occurrence of RpoSnegative strains might result in failed predictions made by predictive models due to their possible higher growth although this remains to be demonstrated in Salmonella. Predictive models must take this into account when considering the limits of accuracy and predicting the survival of Salmonella. Intra-species variability in microbial inactivation kinetics has important implications for food safety. The findings of this study could help to clarify the influence of strain variability in predictive microbiology and in such a way it could be incorporated in microbial risk assessment and in food safety challenge studies by taking into account the behaviour of the most resistant strain under each of the conditions and preparing for the worst case scenario. Furthermore, by knowing the dominant resistance mechanism (e.g. lysine decarboxylase for acid resistance) we could predict the behaviour of the microorganism against the corresponding stress.

\section{ACKNOWLEDGEMENTS}


The work was supported by the Ministry of Higher Education, Malaysia through a scholarship for doctorate studies abroad granted to Wan Zawiah Wan Abdullah. The Salmonella Typhimurium strains were kindly provided by Prof. T.J Humphrey, Institute of Infection and Global Health, University of Liverpool, UK and the remaining strains by Mrs Linda Ward of the Laboratory of Gastrointestinal Infections, Public Health England, Colindale, UK. The help of our colleague Lisa Methven and Ciara Keating (School of Engineering, University of Glasgow) with PCA analysis was invaluable. We dedicate this work to the deceased Dr. Bernard M. Mackey who sadly passed away a few months prior to the completion of this article.

\section{REFERENCES}

1. Álvarez-Ordóñez, A., A. Fernández, A. Bernardo, M. López. 2010. Arginine and lysine decarboxylases and the Acid Tolerance Response of Salmonella Typhimurium. Int. J. Food Microbiol. 136: 278-282.

2. Brooker, D. C., M. E. Lund, and D. J. Blazevic, 1973. Rapid Test for Lysine Decarboxylase activity in Enterobacteriaceae. Appl. Microbiol. 26: 622-623.

3. CDC, 2015. Salmonella. Available at http://www.cdc.gov/salmonella/reportspubs/salmonellaatlas/serotyping-importance.html. Accessed 15 January 2015.

4. Commons, R., J. Dimitriou, and I. Campbell. 2008. Acalculous cholecystitis caused by Salmonella Virchow. ANZ. J. Surg. 78(6): 514

5. De Jonge, R., W. S. Ritmeester, and F. M. van Leusden. 2003. Adaptive responses of Salmonella enterica serovar Typhimurium DT104 and other S. Typhimurium strains and Escherichia coli $\mathrm{O} 157$ to low pH environments. J. Appl. Microbiol. 94: 625-632.

6. Ferenci, T. 2005. Maintaining a healthy SPANC balance through regulatory and mutational adaptation. Mol Microbiol. 57:1-8.

7. Gerstel, U., C. Park, and U. Römling 2003. Complex regulation of $\operatorname{csg} D$ promoter activity by global regulatory proteins. Mol. Microbiol. 49:639-654.

8. Hamilton, S., R. J. Bongaerts, F. Mulholland, B. Cochrane, J. Porter, S. Lucchini, H. M. Lappin- 
Scott, and J. C. Hinton. 2009. The transcriptional programme of Salmonella enterica serovar Typhimurium reveals a key role for tryptophan metabolism in biofilms. BMC Genomics. 10:599. doi: 10.1186/1471-2164-10-599.

9. Hengge, R., 2009. Principles of c-di-GMP signalling in bacteria. Nat. Rev. Microbiol. 7: 263273.

10. Hernández, S.B., Cota, I., Ducret, A., Aussel, L., Casadesús, J., 2012. Adaptation and preadaptation of Salmonella enterica to bile. PLoS Genetics. 8(1). doi.org/10.1371/journal.pgen.1002459.

11. Ibanez-Ruiz, M., V. Robbe-Saule, D. Hermant, S. Labrude, and F. Norel. 2000. Identification of RpoS (sigma S)-regulated genes in Salmonella enterica serovar Typhimurium. J. Bacteriol. 182: $5749-5756$.

12. Jennings, M. E., L. N. Quick, A. Soni, R. R. Davis, K. Crosby, C. M. Ott, C. A. Nickerson, and J. W. Wilson. 2011. Characterization of the Salmonella enterica serovar Typhimurium ydcI gene, which encodes a conserved DNA Binding protein required for full acid stress resistance. $J$. Bacteriol. 193: 2208-2217.

13. Jørgensen, F., S. Leach, S. J. Wilde, A. Davies, G. S. Stewart, and T. J. Humphrey. 2000. Invasiveness in chickens, stress resistance and RpoS status of wild-type Salmonella enterica subsp. enterica serovar Typhimurium definitive type 104 and serovar Enteritidis phage type 4 strains. Microbiol. 146(12): 3227-3235.

14. Karatzas, K. A. G., L. P. Randall, M. Webber, L. J. V. Piddock, T. J. Humphrey, M. J. Woodward, and N. G. Coldham. 2008. Phenotypic and proteomic characterization of multiply antibiotic-resistant variants of Salmonella enterica serovar Typhimurium selected following exposure to disinfectants. Appl. Environ. Microbiol. 74:1508-1516.

15. Kikuchi, Y., O. Kurahashi, T. Nagano, and Y. Kamio. 1998. RpoS-dependent expression of the second lysine decarboxylase gene in Escherichia coli. Biosci. Biotechnol. Biochem. 62: 12671270.

16. Lacroix, F. J. C., A. Cloeckaert, O. Grépinet, C. Pinault, M. Y. Popoff, H. Waxin, and P. Pardon. 1996. Salmonella Typhimurium acrB-like gene: Identification and role in resistance to biliary 
salts and detergents and in murine infection. FEMS Microbiol Lett. 135: 161-167.

17. Lee, Y. H., B. H. Kim, J. H. Kim, W. S. Yoon, S. H. Bang, and Y. K. Park. 2007. CadC has a global translational effect during acid adaptation in Salmonella enterica serovar Typhimurium. J. Bacteriol. 189: 2417-2425.

18. Lianou, A., and K. P. Koutsoumanis. 2011. Effect of the growth environment on the strain variability of Salmonella enterica kinetic behavior. Food Microbiol. 28: 828-837.

19. Lianou, A., and K. P. Koutsoumanis. 2013. Strain variability of the behavior of foodborne bacterial pathogens: A review. Int. J. Food Microbiol. doi:10.1016/j.ijfoodmicro.2013.09.016

20. Lianou, A., Nychas, G.J.E., K. P. Koutsoumanis. 2017. Variability in the adaptive acid tolerance response phenotype of Salmonella enterica strains. Food Microbiol. 62, 99-105.

21. Liu. Z., H. Niu, S. Wu, and R. Huang. 2014. CsgD regulatory network in a bacterial trait-altering biofilm formation. Emerg. Microbes Infect. 3:e1. doi: 10.1038/emi.2014.1.

22. Notley-McRobb, L., T. King, and T. Ferenci. 2002. rpoS mutations and loss of general stress resistance in Escherichia coli populations as a consequence of conflict between competing stress responses. J. Bacteriol. 184, 806-811.

23. O'Toole, G. A., and R. Kolter. 1998. Initiation of biofilm formation in Pseudomonas fluorescens WCS365 proceeds via multiple, convergent signalling pathways: a genetic analysis. Mol. Microbiol. 28:449-461.

24. Prieto, A.I., F. Ramos-Morales, and J. Casadesús. 2006. Repair of DNA damage induced by bile salts in Salmonella enterica. Genetics. 174: 575-584.

25. Prouty, A.M., J. C. van Velkinburgh, and J. S. Gunn. 2002. Salmonella enterica serovar Typhimurium resistance to bile: Identification and characterization of the tolQRA cluster. $J$. Bacteriol. 184: 1270-1276.

26. Soksawatmaekhin, W., A. Kuraishi, K. Sakata, K. Kashiwagi, and K. Igarashi. 2004. Excretion and uptake of cadaverine by $\mathrm{CadB}$ and its physiological functions in Escherichia coli. Mol. Microbiol. 51: 1401-1412.

27. Spector, M. P., and W. J. Kenyon. 2012. Resistance and survival strategies of Salmonella 
enterica to environmental stresses. Food Res. Int. 45: 455-481.

28. Viala, J. P., M., S. Méresse, B. Pocachard, A. A. Guilhon, L. Aussel, and F. Barras. 2011. Sensing and adaptation to low $\mathrm{pH}$ mediated by inducible amino acid decarboxylases in Salmonella. PLoS One. 6(7):e22397.

29. Vijayakumar, S. R. V., M. G. Kirchhof, C. L. Patten, and H. E. Schellhorn. 2004. RpoSregulated genes of Escherichia coli identified by random lacZ fusion mutagenesis. J. Bacteriol. 186: 8499-8507.

30. Waldner, L.L., K. D. Mackenzie, W. Köster, A. P. White, W. Kö, A. P. White, W. Köster, and A. P. White. 2012. From Exit to Entry: Long-term Survival and Transmission of Salmonella. Pathogens 1(2): 128-155.

31. Wang, H., Jiang, Y., Liu, X., Qian, W., Xu, X., Zhou, G., 2016. Behavior variability of Salmonella enterica isolates from meat-related sources. LWT - Food Sci. Technol. 73, 375-382. doi:10.1016/j.lwt.2016.06.036

32. White, A. P., and M. G. Surette. 2006. Comparative Genetics of the rdar morphotype in Salmonella. J. Bacteriol. 188:8395-8406.

33. WHO, 2013. Salmonella (non-typhoidal). Fact sheet $\mathrm{N}^{\circ} 139$. Available at http://www.who.int/mediacentre/factsheets/fs139/en/.Accessed 15 Jan 2015.

34. Winfield, M. D., and E. A. Groisman. 2003. Role of non-host evironments in the lifestyles of Salmonella and Escherichia coli. Appl. Environ. Microbiol. 69:3687-3694.

35. Zambrano, M. M., D. A. Siegele, M. Almiron, A. Tormo, and R. Kolter. 1993. Microbial competition: Escherichia coli mutants that take over stationary phase cultures. Science. 259:1757-1760. 
Table 1. Salmonella enterica serovars used in this study

\begin{tabular}{llll}
\hline Serovar & $\begin{array}{l}\text { Strain } \\
\text { number }\end{array}$ & $\begin{array}{l}\text { Isolated } \\
\text { from }\end{array}$ & Source or reference \\
\hline Anatum & S180332 & Dried Food & $\begin{array}{l}\text { Laboratory of Enteric Pathogens, } \\
\text { Public Health Laboratory Service. } \\
\text { Colindale, London NW9 5EQ1 }\end{array}$ \\
& & & \\
Heidelberg & S172457 & Chicken & \\
Montevideo & S182788 & Pet food & \\
Newport & S186166 & Peppercorns & \\
Enteritidis & P518466 & Human & \\
Enteritidis & P518496 & Human & \\
Virchow & P518634 & Pet food & \\
Typhimurium DT 104 & Strain 10 & Human & Jorgensen et. al. (2000) \\
Typhimurium DT 104 & Strain 30 & Bovine & \\
\hline
\end{tabular}

${ }^{1}$ Now Public Health England, Microbiological Services 
Table 2. Colony morphology of representative strains of Salmonella enterica serovars

\begin{tabular}{|c|c|c|}
\hline Serovar & $\begin{array}{l}\text { Appearance } \\
\text { on MBGA }\end{array}$ & Appearance on Congo Red agar \\
\hline $\begin{array}{l}\text { Anatum } \\
\text { Montevideo }\end{array}$ & $\begin{array}{l}\text { convoluted } \\
\text { convoluted }\end{array}$ & $\begin{array}{l}\text { rdar, convoluted } \\
\text { rdar, convoluted }\end{array}$ \\
\hline Enteritidis 496 & convoluted & rdar ,convoluted \\
\hline Virchow & convoluted & rdar, convoluted \\
\hline Typhimurium 30 & convoluted & rdar, convoluted \\
\hline Newport & convoluted & $\begin{array}{l}\text { Initially pink, weakly convoluted, developing the rdar } \\
\text { convoluted phenotype after } 5 \text { days }\end{array}$ \\
\hline Enteritidis 466 & smooth & $\begin{array}{l}\text { Initially pink, smooth, developing pimples on surface } \\
\text { after } 5 \text { days }\end{array}$ \\
\hline Hadar & smooth & Pink, smooth \\
\hline Typhimurium 10 & smooth & White/pale yellow, smooth circular \\
\hline Heidelberg & smooth & White/pale yellow, smooth circular \\
\hline
\end{tabular}


Table 3. RpoS phenotypes of Salmonella enterica serovars

\begin{tabular}{lccc}
\hline Serovar & Glycogen & Catalase & RpoS phenotype \\
\hline Anatum & + & + & + \\
Enteritidis 466 & + & + & + \\
Enteritidis 496 & + & + & + \\
Hadar & + & + & + \\
Heidelberg & - & - & - \\
Montevideo & + & + & + \\
Newport & + & + & + \\
Typhimurium 10 & - & - & - \\
Typhimurium 30 & + & + & + \\
Virchow & + & + & + \\
\hline
\end{tabular}


Table 4. Rank order in motility of Salmonella enterica strains averaged over three incubation temperatures ${ }^{a}$

\begin{tabular}{lc}
\hline \multicolumn{1}{c}{ Serovar } & Rank Order \\
\hline Anatum & $1^{\mathrm{b}}$ \\
Enteritidis 466 & 1 \\
Enteritidis 496 & 3 \\
Hadar & 4 \\
Heidelberg & 5 \\
Montevideo & 6 \\
Newport & 6 \\
Typhimurium 10 & 8 \\
Typhimurium 30 & 9 \\
Virchow & 10
\end{tabular}

a Motility was assessed at $15^{\circ} \mathrm{C}, 30^{\circ} \mathrm{C}$ and $37^{\circ} \mathrm{C}$

b1 = most motile 
Table 5. Optical densities of stained biofilms of Salmonella enterica strains after growth in polystyrene microwell plates

\begin{tabular}{llll}
\hline Serovar & $\begin{array}{l}\text { Optical } \\
\text { density }\end{array}$ & $\begin{array}{l}\text { Standard } \\
\text { deviation }\end{array}$ & Rank \\
\hline Montevideo & 0.60 & 0.00 & 1 \\
\hline Virchow & 0.49 & 0.61 & 2 \\
\hline Anatum & 0.40 & 0.12 & 3 \\
\hline Typhimurium 30 & 0.37 & 0.27 & 4 \\
\hline Enteritidis 496 & 0.27 & 0.08 & 5 \\
\hline Hadar & 0.22 & 0.11 & 6 \\
\hline Newport & 0.20 & 0.16 & 7 \\
\hline Typhimurium 10 & 0.10 & 0.40 & 8 \\
\hline Heidelberg & 0.07 & 0.08 & 9 \\
\hline Enteritidis 466 & 0.04 & 0.19 & 10 \\
\hline
\end{tabular}


Table 6. Growth of Salmonella enterica strains

in LB medium containing sodium deoxycholate

\begin{tabular}{|c|c|c|c|c|c|c|}
\hline \multirow[b]{2}{*}{ Serovar } & \multicolumn{6}{|c|}{$\begin{array}{l}\text { Concentration of sodium deoxycholate } \\
\qquad(\% \mathrm{w} / \mathrm{v})\end{array}$} \\
\hline & 0.5 & 1.0 & 2.0 & 3.0 & 4.0 & 5.0 \\
\hline Hadar & + & + & + & + & + & + \\
\hline Anatum & + & + & + & + & + & + \\
\hline Montevideo & + & + & + & + & + & \pm \\
\hline Newport & + & + & + & + & + & \pm \\
\hline Enteritidis 466 & + & + & + & + & \pm & + \\
\hline Enteritidis 496 & + & + & + & + & \pm & + \\
\hline Heidelberg & + & + & + & + & + & - \\
\hline Typhimurium 30 & + & + & + & + & + & \pm \\
\hline Typhimurium 10 & + & + & + & \pm & + & + \\
\hline Virchow & + & + & + & \pm & - & - \\
\hline
\end{tabular}

$\overline{1+\text { Growth in both duplicates, } \pm \text { growth in one duplicate, - no growth }}$ 
Table 7. Lag ratios ${ }^{a}$ for Salmonella serovars grown in LB containing sodium deoxycholate

\begin{tabular}{lccccccc}
\hline & \multicolumn{6}{c}{ Concentration of sodium deoxycholate } \\
\hline Serovar & \multicolumn{3}{c}{$\mathbf{0 . 5 \%}$} & \multicolumn{1}{c}{$\mathbf{1 . 0 \%}$} & $\mathbf{2 . 0} \%$ & Mean \\
Anatum & 1.3 & 1.0 & 1.1 & 1.2 & 1.1 & 1.3 & 1.1 \\
Enteritidis 466 & 0.9 & 0.7 & 0.8 & 0.7 & 0.9 & 1.0 & 0.8 \\
Enteritidis 496 & 0.8 & 0.8 & 1.7 & 1.5 & 1.2 & 1.1 & 1.2 \\
Hadar & 0.9 & 0.9 & 0.8 & 0.9 & 0.8 & 0.8 & 0.8 \\
Heidelberg & 0.9 & 0.7 & 0.9 & 0.8 & 1.0 & 1.0 & 0.9 \\
Montevideo & 0.7 & 0.7 & 1.0 & 0.9 & 1.1 & 1.0 & 0.9 \\
Newport & 0.8 & 0.7 & 0.6 & 0.7 & 0.4 & 0.5 & 0.6 \\
Typhimurium 10 & 0.7 & 2.0 & 0.5 & 0.5 & 1.1 & 1.0 & 0.9 \\
Typhimurium 30 & 0.8 & 0.7 & 0.5 & 0.7 & 0.9 & 0.8 & 0.7 \\
Virchow & 2.3 & 2.0 & 2.0 & 2.1 & 1.6 & 1.5 & 1.9 \\
\hline Mean lag all & $245 \pm 118$ & $431 \pm 206$ & $621 \pm 174$ & \\
serovars \pm SD(min) & 245
\end{tabular}

a Lag time of individual serovar/mean lag time all serovars 
Table 8. Rank order of resistance to adverse conditions among Salmonella serovars

\begin{tabular}{lcccc}
\hline Serovar & $\begin{array}{c}\text { Acetic } \\
\text { acid pH } \\
\mathbf{4 . 0}\end{array}$ & $\begin{array}{c}\text { Sodium } \\
\text { chloride } \\
\mathbf{( 9 \% )}\end{array}$ & $\begin{array}{c}\text { Acetic } \\
\text { acid } \\
\text { plus } \\
\text { salt }\end{array}$ & $\begin{array}{c}\text { Overall } \\
\text { rank }\end{array}$ \\
\hline Hadar & 1 & 1 & 4 & 1 \\
Enteritidis 496 & 1 & 5 & 2 & 2 \\
Enteritidis 466 & 1 & 7 & 2 & 3 \\
Virchow & 7 & 2 & 1 & 3 \\
Typhimurium 30 & 6 & 3 & 5 & 5 \\
Newport & 4 & 5 & 6 & 6 \\
Anatum & 4 & 4 & 8 & 7 \\
Montevideo & 8 & 8 & 7 & 8 \\
Heidelberg & 9 & 9 & 9 & 9 \\
Typhimurium 10 & 9 & 9 & 10 & 10 \\
\hline
\end{tabular}




\section{LEGENDS TO FIGURES}

Fig 1. Colony morphology on Congo Red agar. A. Smooth colony of Salmonella serovar Typhimurium 10; B, convoluted colony of Salmonella serovar Typhimurium 30.

Fig 2. The rpoS gene of $S$. Heidelberg used in this study contained an insertion of a T (indicated with a grey oval) at nucleotide number 640 , causing a +1 frameshift mutation that alters completely the protein sequence after codon 214 most probably resulting in a non-functional protein. The sequence of $S$. Heidelberg str. B182 RpoS protein and rpoS gene are shown as a reference of a representative S. Heidelberg strain.

Fig 3. Motility of Salmonella serovars on agar plate of semi-solid MTM. Strains were inoculated at the centre of the plate and incubated at $30^{\circ} \mathrm{C}$. The distance cells migrated from the centre was measured after $12 \mathrm{~h}$. Asterisk indicates statistically significant $(\mathrm{P}<0.01)$ difference with all other isolates.

Fig 4. Biofilm formation in Salmonella serovars. Cells were grown in Low Salt Luria Broth incubated at $20^{\circ} \mathrm{C}$ for $4 \mathrm{~d}$ and biofilm was assessed before and after the removal of the culture.

Fig 5. Effect of sodium deoxycholate on the growth kinetics of Salmonella serovar Hadar. Salmonella Hadar was inoculated into LB containing graded concentrations of sodium deoxycholate (DOC) and optical density was measured at intervals in a microplate reader. Control no DOC $(\mathbf{X})$; 0.5\% DOC $(\bullet) ; 1.0 \% \operatorname{DOC}(\mathrm{O}) ; 2.0 \% \operatorname{DOC}(\boldsymbol{\Delta}) ; 3 \% \operatorname{DOC}(\Delta) ; 4 \% \operatorname{DOC}(\boldsymbol{\square}) ; 5 \% \operatorname{DOC}(\square)$. 
Fig 6. Survival kinetics of Salmonella serovars under mild acid conditions. Salmonella strains were grown for $18 \mathrm{~h}$ in TSB and subsequently diluted 100-fold into NB containing $20 \mathrm{mM}$ sodium acetate $\mathrm{pH}$ 4.5. Viable counts were determined at intervals during incubation at $25^{\circ} \mathrm{C}$.

Fig 7. Survival of Salmonella serovars under mild stress conditions. Strains were grown in TSB for $18 \mathrm{~h}$, subsequently diluted 100-fold into NB containing (A) $20 \mathrm{mM}$ sodium acetate $\mathrm{pH} 4.0$, (B) $9.0 \%$ $\mathrm{NaCl}$, (C) $20 \mathrm{mM}$ sodium acetate $\mathrm{pH} 4.0$ plus $9 \% \mathrm{NaCl}$. Viable counts were determined at intervals during incubation at $25^{\circ} \mathrm{C}$ and the time for a 1000 -fold (3-log) reduction calculated.

Fig 8. pH increase elicited by the lysine decarboxylase activity of Salmonella serovars in lysine decarboxylase medium.

Fig 9. Correlation between lysine decarboxylase activity and acid resistance among different serovars. Lysine decarboxylase activity was measured as the time taken for the $\mathrm{pH}$ of the suspending medium to increase by 0.5 units. Acid resistance was represented as the time taken for viable numbers to decrease 1000 -fold in the presence of $20 \mathrm{mM}$ lactic acid at $\mathrm{pH} 3.5$ (A) or $20 \mathrm{mM}$ acetic acid at $\mathrm{pH}$ 4.0 (B). The two points at lower right are the two RpoS-negative serovars Typhimurium 10 and Heidelberg.

Fig 10. PCA plot from strain phenotypic analysis including all data (A) and only data on stress resistance (B). Variation explained by PC1/PC2. 